\title{
1 Stable hydrophilic poly(dimethylsiloxane) via glycan surface 2 functionalization
}

3 Leticia Esteban-Tejeda, ${ }^{a b}$ Thomas Duff, ${ }^{a b}$ Guido Ciapetti, ${ }^{a}$ M. Daniela Angione, ${ }^{a b}$ Adam

4 Myles, ${ }^{a}$ Joana M. Vasconcelos, ${ }^{a}$ Eoin M. Scanlan, ${ }^{a{ }^{*}}$ Paula E. Colavita ${ }^{a b *}$

$5 \quad$ aSchool of Chemistry, Trinity College Dublin, Dublin 2, Ireland.

$6 \quad{ }^{b}$ CRANN and AMBER Research Centres, Trinity College Dublin, Dublin 2, Ireland

$7 \quad$ * E-mail: eoin.scanlan@tcd.ie; colavitp@tcd.ie

\section{Abstract}

Polydimethylsiloxane (PDMS) is an extremely important and versatile polymeric material for biomedical and microfluidic devices due to a range of desirable properties. Control of the hydrophilicity of PDMS surfaces is of significant interest due to the potential for developing surfaces with tunable protein adsorption or cell adhesion properties. We report the formation of stable hydrophilic PDMS surfaces by covalent modification with glycans via aryldiazonium chemistry. The PDMS surface was modified by a two step-process including an activation of the PDMS surface, followed by reaction with aryldiazonium glycosides in aqueous solution. The functionalized PDMS was characterized by atomic force microscopy, infrared and X-ray photoelectron spectroscopy, water contact angle measurements and fluorescence microscopy. Our results demonstrate that glycans immobilized via this methodology have the dual function of imparting hydrophilicity and stabilizing the modified surface against hydrophobic recovery. Importantly, the presentation of thus immobilized glycosides makes them available to specific lectin-glycan binding interactions at the polymer-solution interface while, in the absence of specific binding interactions, leads to a reduction in albumin adsorption. This approach provides a novel and efficient route to stable hydrophilic PDMS surfaces with a broad range of applications. 


\section{1. Introduction}

Polydimethylsiloxane (PDMS) is a biomaterial extensively used in medicine for making catheters, stents [1,2], ocular lenses and pacemaker encapsulants [3]. More recently it has found application in the fabrication of microfluidic chips [4-7]. Despite the many advantages of PDMS, its high hydrophobicity and low surface energy often represent a challenge for controlling sealing, adhesion and flow in microfluidics, as well as for supporting cell adhesion and proliferation in bioanalytical and bioMEMS applications.

To make PDMS more hydrophilic, its surface can be modified through various processes, most notably via dry oxidation using plasma [8,9], corona discharge [10] and UV/ozone [11] treatments. These methods can effectively increase hydrophilicity however they are often difficult to integrate into microfabrication processes due to potential damage to other components and materials. Oxidation must be carried out under carefully controlled conditions in order to avoid severe oxidative damage and loss of mechanical properties [4]; furthermore, the above processes are not applicable to the modification of internal surfaces in pre-assembled channels and devices. Importantly, oxidized PDMS surfaces exhibit hydrophobic recovery, a process by which shorter and relatively mobile PDMS chains diffuse through the polymer and rearrange at the surface so as to lower surface free energy [12]. Recovery can take place over relatively short time scales, for instance, it has been reported that the water contact angle can increase more than $50^{\circ}$ within $2 \mathrm{~h}$ after PDMS oxidation [12-20]. The rate of recovery can be decreased by keeping PDMS surfaces in contact with water or polar solvents [21]. Some authors have also proposed a two-step extraction/oxidation process to reduce the concentration of mobile PDMS oligomers: in the first step the unreacted oligomers are removed from the bulk by extraction using different organic solvents, while in the second step the PDMS is oxidized by air plasma [22].

An alternative strategy for preparing hydrophilic PDMS surfaces involves the use of covalent surface functionalization following oxidative treatment. Previous literature examples show that 
oxidation, followed by covalent derivatization with bulky hydrophilic groups is a viable route for creating hydrophilic PDMS surfaces with enhanced stability. Donzel et al., reported on the use of oxidative treatments followed by derivatization with poly(ethylene glycols) (PEGs) as a strategy [23] to reduce hydrophobic recovery. Other chemical functionalities explored for the same purpose are aminopropyltriethoxysilane (APTES) [4], UV grafted polymers [14] and chemically vapour deposited polymers [17], among others. However, most of the methods explored thus far are not applicable to the modification of internal surfaces.

The use of solution-based flow methods can overcome this problem and expand the applications of PDMS surface modifications to complex integrated devices. Herein, we describe a novel strategy for the modification of PDMS surfaces based on a two-step solution protocol; a first step of PDMS activation and a second step of functionalization with carbohydrates via aryldiazonium chemistry. Recent work from our group has demonstrated that aryldiazonium chemistry can be employed for the direct modification of polymers such as polyethersulfone (PES), which has phenyl groups in its backbone [24]. In the current work we report on the use of a mild surface activation step to generate sites that are reactive towards aryldiazonium groups, thus expanding the applications of this chemistry to polymers with relatively inert surfaces. Our results show that glycans immobilized via this methodology have the dual role of imparting hydrophilicity and stabilizing the modified surface against hydrophobic recovery.

\section{Experimental Section}

Chemicals and Materials. Polydimethylsiloxane (PDMS) sheets Class VI were purchased from Goodfellow; sodium hypochlorite (bleach), sodium hydroxide, potassium hydroxide, phosphate buffered saline buffer (0.010 M PBS, pH 7.4), sodium nitrite and fluoroboric acid were purchased from Sigma Aldrich. Lectin Concanavalin A (ConA) and Bovine Serum Albumin (BSA) conjugates with Alexa Fluor 647 were purchased from Biosciences. Lectin ConA and peanut agglutinin from Arachis Hypogaea (PNA)) conjugates with fluorescein isothiocyanate (FITC) were purchased from Sigma Aldrich. 4-Aminophenyl glycosides bearing mannose 
$(\mathrm{ManOPh})$ and lactose $(\mathrm{LacOPh})$, whose molecular structure is shown in Scheme 1, were synthesized as previously described [24, 25].

PDMS Modification. Activation of PDMS surfaces was carried out using two different methods: i) treatment with a $0.5 \%$ bleach in basic aqueous solution ( $\mathrm{KOH} 1 \%$ and $\mathrm{NaOH} 1 \%)$, referred to as $\mathrm{BB}$ from here onwards, for $60 \mathrm{~min}$ in total, while changing the BB solution every 20 min with a fresh aliquot; ii) exposure to UV light in air over different time intervals (10-30 min) using a mercury grid lamp (UVP). The lamp had a peak $254 \mathrm{~nm}$ irradiance of approximately $6 \mathrm{~mW} \mathrm{~cm}^{-2}$ at the sample distance [26], and an emission at $185 \mathrm{~nm}$ rated at $3 \%$ of the $254 \mathrm{~nm}$ line by the manufacturer. After activation, functionalization of the PDMS surface pre-activated via (i) or (ii), was carried out via incubation in freshly prepared $1.0 \mathrm{mM}$ solutions of aryldiazonium cations of $\mathrm{ManOPh}$ and $\mathrm{LacOPh}$ generated in situ from the aminophenyl precursor compound, following the method described by Jayasundara et al. [25].

Surface Characterization. Attenuated Total Reflection Fourier Transform Infrared spectroscopy (ATR-FTIR) spectroscopy measurements were collected using a Perkin-Elmer GX spectrometer, at $4 \mathrm{~cm}^{-1}$ resolution. X-ray photoelectron spectroscopy was performed on a VG Scientific ESCAlab Mk II system $\left(<2 \times 10^{-8}\right.$ mbar), using an Al $\mathrm{K} \alpha$ source $(1486.6 \mathrm{eV})$; pass energy was set at $200 \mathrm{eV}$ for survey spectra and $20 \mathrm{eV}$ for high resolution spectra. Spectra were collected with an emission current of $100 \mathrm{~mA}$ at $50 \%$ of normal operating conditions (200 mA) to minimize sample damage. Peaks were referenced to the $\mathrm{C} 1 \mathrm{~s}$ core energy to correct for charging prior to analysis using CasaXPS software. Atomic force microscopy (AFM, Asylum Research) was carried out in tapping mode and analysis of surface topography was carried out using image analysis software (Gwyddion) from $512 \times 512$ lines images. Static contact angles were measured on a commercial system (FTA) in air, using $20 \mu \mathrm{L}$ droplets.

Biomolecule-PDMS binding studies. PDMS samples were incubated for $1 \mathrm{~h}$ (Alexa Fluor conjugates) or $2 \mathrm{~h}$ (FITC conjugates) in a $0.5 \mathrm{mg} / \mathrm{mL}$ lectin solution in PBS buffer at $\mathrm{pH} 7.4$ including $0.1 \mathrm{mM} \mathrm{CaCl}{ }_{2}$ and $\mathrm{MgCl}_{2}$. For protein adsorption studies, PDMS samples were 
incubated for $1 \mathrm{~h}$ in a $0.2 \mathrm{mg} / \mathrm{mL}$ BSA solution in PBS buffer at $\mathrm{pH}$ 7.4. After incubation, surfaces were washed with PBS solution to remove unbound protein prior to imaging. Fluorescence images were acquired using an Olympus BX51 inverted microscope with a cellSense digital image processing software. Images were acquired with a cube filter set having a $635 \mathrm{~nm}$ EdgeBasic long-pass edge filter, a single-edge dichroic beamsplitter filter at $649 \mathrm{~nm}$ and a barrier filter at $620-640 \mathrm{~nm}$. Analysis of emission intensities was carried out in triplicate using Image J software.

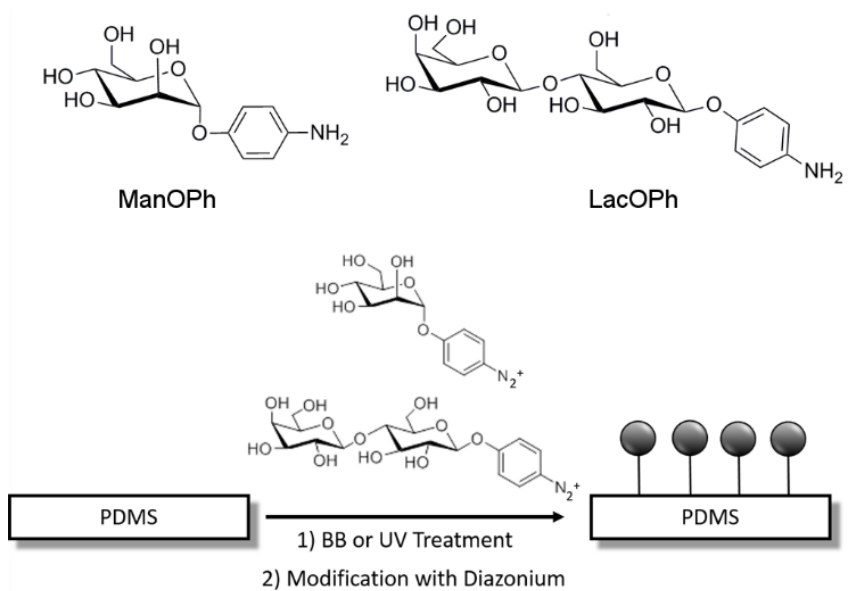

Scheme 1. 4-aminophenyl mannoside ( $\mathrm{ManOPh})$ and lactoside ( $\mathrm{LacOPh})$ used for functionalization (top); activation and functionalization protocols used for the modification of PDMS (bottom).

\section{Results and discussion}

The functionalisation method consisted of a two-step process illustrated in Scheme 1. The first step consists in an activation pre-treatment of PDMS surfaces via exposure to a solution of bleach and base (BB), or via exposure to UV in air. BB solutions are known to be good oxidants [27-33] and their elevated hydroxide concentration can also chemically degrade PDMS via well known base-catalyzed depolymerization reactions [34-37]. To our knowledge, however, BB solutions have not been previously investigated as a means of activating PDMS for functionalization. UV exposure in air, on the other hand, is frequently used for the oxidative modification of PDMS [10, 38-43] and was used as a term of comparison for our studies. 
126 Following activation, surfaces were immersed in a solution of the aryldiazonium cation of the

127 phenylglycosides, using previously reported conditions [25].

\section{Effect of activation pre-treatments.}

129 The effect of UV and BB pre-treatment on the surface morphology was determined by AFM 130 imaging in tapping mode. Analysis of neat PDMS samples yielded a root-mean-square 131 roughness (rms) of $3.7 \pm 0.6 \mathrm{~nm}$. After BB-pretreatment and UV exposure for $30 \mathrm{~min}$, the rms values were found to be $3.7 \pm 0.7 \mathrm{~nm}$ and $3.7 \pm 0.4 \mathrm{~nm}$, respectively, thus indicating that the pre-treatments do not have a statistically significant effect on PDMS roughness.

XPS analysis was carried out to investigate chemical changes caused by BB and UV treatment on PDMS surfaces. High resolution spectra in the Si 2p, C 1s and O 1s regions of neat and treated PDMS samples are shown in Figure 1. Spectra were used to calculate peak area ratios which, corrected by relative sensitivity factors $(\mathrm{RSF} C=1, \mathrm{O}=2.93, \mathrm{Si}=0.82$ ), yielded the elemental atomic ratios summarised in Table 1. The neat PDMS yielded O/C and Si/O values of 0.6 and 1.0, close to those expected from the stoichiometry of the polymer, and in good agreement with previous literature results $[6,38,39,43-45]$. UV treatments led to a slight increase in the surface $\mathrm{O} / \mathrm{C}$ ratio to 0.7 after 30 min exposure to UV light; this is consistent with an increase in surface density of silanol groups which has been reported to result from UV/ozone oxidative treatments in air [43]. Treatment with BB solution was found to result in a slight reduction in oxygen content and a surface enrichment of $\mathrm{Si}$ atoms $(\mathrm{Si} / \mathrm{O}=1.2)$; a modest reduction in the $\mathrm{Si} 2 \mathrm{p}$ peak width is also observable, and suggests greater homogeneity of $\mathrm{Si}$ sites at the activated PDMS surface. This result suggests that treatment with BB solutions does not yield increased concentrations of surface bound O-containing groups as in the case of the UV treatment. Hydroxide ions are known to catalyze the depolymerization of PDMS which results in chain cleavage, cyclicized products and formation of insoluble and soluble silanolates

150 [34-37]. In particular, the loss of soluble disilanolates should lead to a reduction in surface 
in the $\mathrm{Si} 2 \mathrm{p}$ peak width is also supportive of depolymerization via cyclization. Insoluble

153 silanolates with high molecular weight are known to remain at the surface of base-treated

154 PDMS;[46, 47] however, it is challenging to discriminate the presence of deprotonated 155 silanolates from that of silanols via XPS, as deprotonation does not alter the C:Si:O ratio of the polymer surface.

Si $2 p$

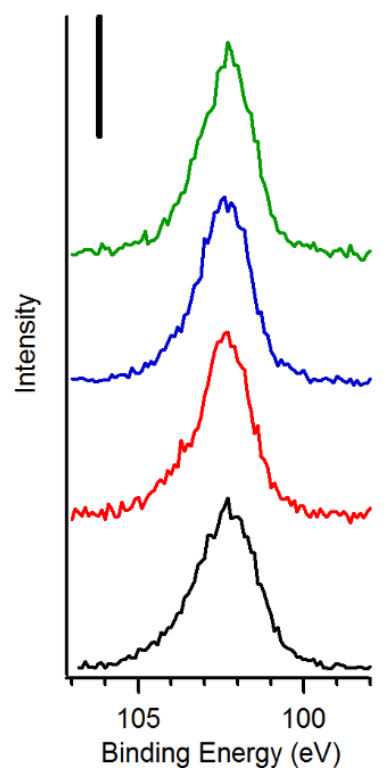

C 1s

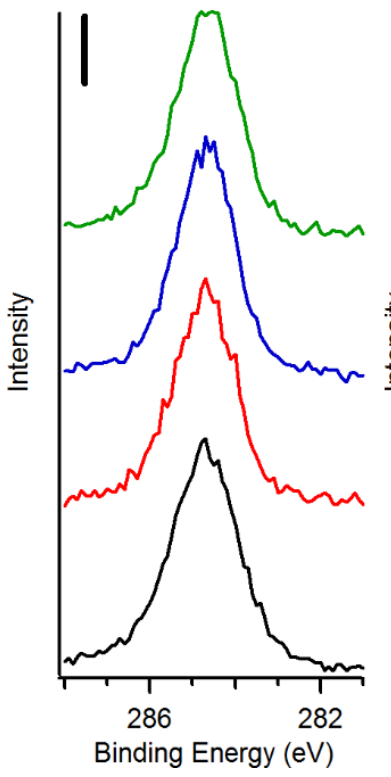

$01 \mathrm{~s}$

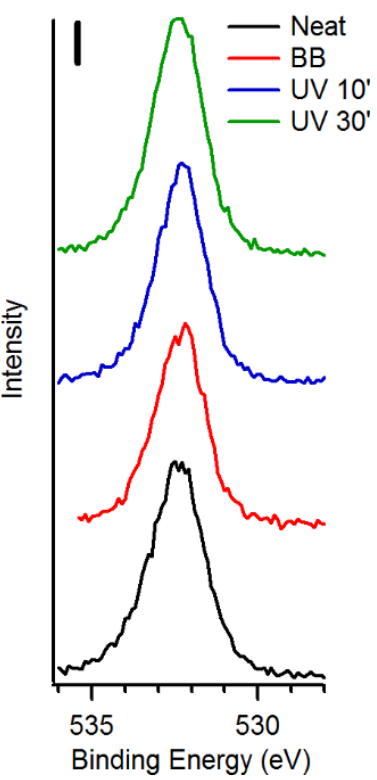

159

160

161

162

163

Figure 1. XPS spectra in the Si $2 p, C$ s and O 1 s regions of neat PDMS, BB-treated PDMS, and PDMS exposed for 10 and 30 min to UV light. Spectra were shifted to correct for charging using the $\mathrm{C} 1 \mathrm{~s}$ peak position of $284.6 \mathrm{eV}$ as reference; peaks are normalized by the height of the $\mathrm{C} 1 \mathrm{~s}$ to facilitate comparison. The scalebar is equivalent to 500 counts in all three regions.

\begin{tabular}{lcccc}
\hline Material & C\% & O\% & Si\% & WCA \\
\hline PDMS neat & 45 & 27 & 28 & $112.0 \pm 0.5$ \\
PDMS BB & 45 & 24 & 31 & $100.7 \pm 0.6$ \\
PDMS UV 10, & 45 & 26 & 29 & $92.3 \pm 0.7$ \\
PDMS UV 30, & 43 & 28 & 29 & $84.0 \pm 0.3$ \\
\hline
\end{tabular}

Table 1. Atomic \% composition obtained from XPS, and water contact angle of neat PDMS, PDMS pre-treated with BB solution and PDMS pre-treated with UV light after 10 min and 30 min exposure. 
To investigate the effect that the pre-treatment steps have on surface hydrophilicity, we carried out studies using both infrared spectroscopy (ATR-FTIR) and water contact angle measurements. Figure 2a shows IR spectra of neat and treated PDMS in the $1800-1300 \mathrm{~cm}^{-1}$ region collected under identical conditions; all spectra have been normalized by the nearest peak at $1412 \mathrm{~cm}^{-1}$, assigned to the asymmetric bending of $-\mathrm{CH}_{3}$ groups [48]. Spectra display a broad peak between $1650-1600 \mathrm{~cm}^{-1}$ which can be assigned to the bending mode of water, $\beta(\mathrm{HOH})$ adsorbed at the polymer surface [24, 49]. Exposure to UV over either 10 or 30 min leads to an increase in the integrated area of the $\beta(\mathrm{HOH})$ peak and a shift to higher wavenumbers. This behaviour is typical of an increase in adsorbed water at polymer surfaces, as previously reported in the literature [49]. Treatment with BB shows only a small increase in the intensity of the $\beta(\mathrm{HOH})$ peak.

Figure $2 \mathrm{~b}$ shows the IR spectra of neat and treated PDMS in the $4000-2900 \mathrm{~cm}^{-1}$ region. The figure shows a broad peak centered at $\sim 3350 \mathrm{~cm}^{-1}$ that arises from $\mathrm{O}-\mathrm{H}$ stretching modes of silanol groups and adsorbed molecular water; the peak is convoluted with the $\mathrm{C}-\mathrm{H}$ stretching vibrations of the methyl groups in PDMS at 2963 and $2906 \mathrm{~cm}^{-1}$, in good agreement with the literature [43]. The $\mathrm{C}-\mathrm{H}$ stretching absorbances are not shown in their entirety to more clearly compare the $\mathrm{O}-\mathrm{H}$ stretching contributions. The overall intensity of the $\mathrm{O}-\mathrm{H}$ stretching peak follows a similar trend to that observed for the bending modes: neat PDMS and BB-treated PDMS show the lowest peak intensities, while UV treatment, after either 10 or $30 \mathrm{~min}$, leads to a visible increase in the intensity of the peak indicating a higher surface density of silanol and/or molecular water, in agreement with previous reports [43]. It is interesting to note that BB treated PDMS possesses the lowest intensity in this range among surfaces examined; this suggests that BB treatment does not lead to an increase in silanol groups, in agreement with XPS results. A reduction in $\mathrm{O}-\mathrm{H}$ stretching intensity remains consistent however with formation of silanolates via exposure to BB solutions.

Water contact angle measurements using the sessile drop method were also used to determine the effect of surface treatments on wetting properties and results are summarized in Table 1 . The 
water contact angle of neat PDMS was found to be $112^{\circ}$ in good agreement with literature values $[6,10]$. After treatment with $\mathrm{BB}$ solution the water $\mathrm{CA}$ decreased to $100.7^{\circ}$; a greater decrease was obtained via UV treatment which yielded water CAs of $92.3^{\circ}$ and $84.8^{\circ}$ after 10 and $30 \mathrm{~min}$ exposure, respectively. These results confirm the trends observed via infrared spectroscopy and support the correlation between the surface concentration of oxidised groups and surface wetting behaviour. In summary, XPS, FTIR and WCA results indicate that UV treatments lead to surface oxidation and an increase in surface hydrophilicity, while the BB activation process does not lead to significant oxidation and correspondingly the change in WCA is much smaller; however, BB activation is likely to result in surface depolymerization of PDMS as suggested by XPS data.

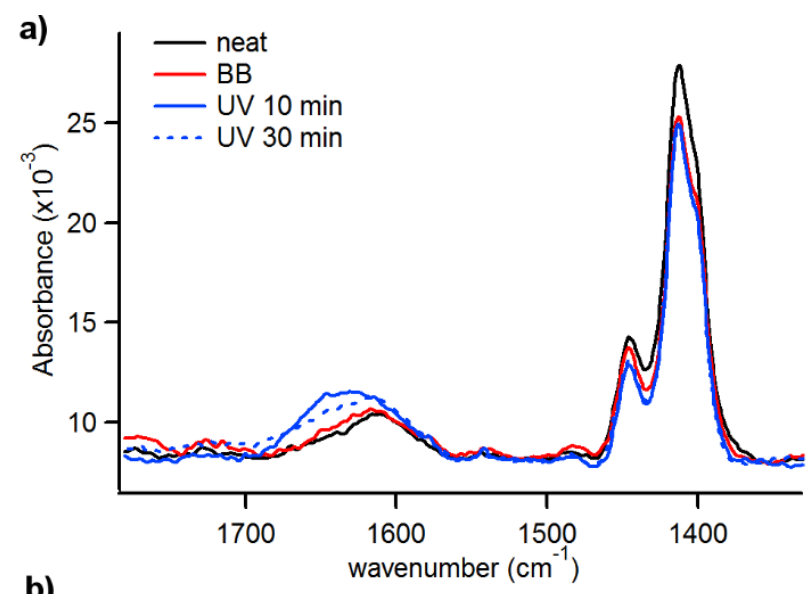

b)

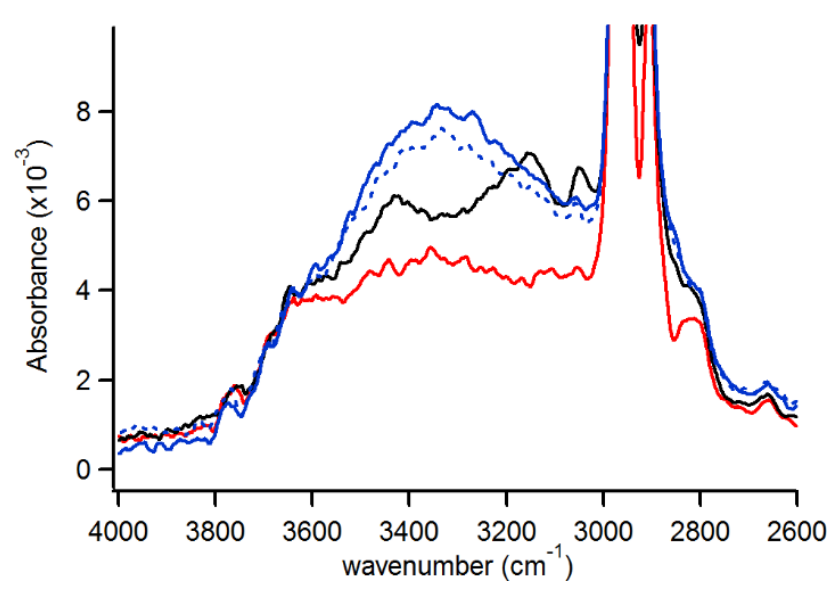

Figure 2. ATR-FT-IR of native PDMS, pretreated PDMS with BB solution, pretreated PDMS with UV light within $10 \mathrm{~min}$ and $30 \mathrm{~min}$ exposure in the $\mathrm{HOH}$ bending (a) and $\mathrm{OH}$ stretching regions (b). 
211

212

213

PDMS surfaces treated via BB and UV exposure were functionalised via immersion in freshly prepared solutions of glycosides of aryldiazonium salts. After modification, surfaces were rinsed and dried; binding studies were then carried out to establish that glycans are immobilized via the protocols described in Scheme 1. Functionalization was first confirmed via ConA binding experiments on mannose-functionalized PDMS surfaces; specific binding interactions between ConA and mannose are well established and can be used to confirm the presence of surface-bound mannosides [50, 51]. Samples were incubated for $1 \mathrm{~h}$ in a solution of fluorescently labeled ConA and rinsed afterwards with PBS prior to imaging. Figure 3a shows fluorescence microscopy images after ConA incubation of PDMS surfaces corresponding to: neat PDMS, pre-treated PDMS and mannose-functionalized PDMS; Figure 3b summarises average emission intensity values obtained for samples as shown in Figure 3a. Neat PDMS surfaces were found to yield low emissions, thus indicating that the amount of ConA unspecifically bound at the PDMS surface is very low. This is consistent with the low surface free energy of PDMS surfaces, which is reported at $23 \mathrm{~mJ} \mathrm{~m}^{-2}$ and that is proposed to make this polymer one of the best materials in terms of protein rejection, placing it close to the minimum in the Baier curve $[52,53]$. After undergoing BB or UV oxidative treatments, an increase in emission is observed indicating greater unspecific adsorption of ConA at oxidized vs. neat PDMS surfaces. After modification with ManOPh groups (PDMS-Man), a further increase in emission is observed after both oxidative pre-treatments, thus suggesting that aryldiazonium reaction increases ConA binding, as expected after immobilization of mannosides [24, 25]. Control experiments indicate that no significant ConA binding occurs on neat PDMS surfaces after exposure to aryldiazonium mannosides in the absence of pre-treatment. This indicates that the BB or UV pre-treatment steps are necessary in order to carry out aryldiazonium modification of PDMS. Although the mechanism of the reaction with PDMS surfaces is not studied in detail in this manuscript, aryldiazonium cations are highly reactive species that are known to cross-couple to nucleophilic groups, including hydroxyl groups. It is 

increase in surface hydroxyl (silanol) groups. Therefore, we propose that the role of BB treatments is to enhance the reactivity of surface nucleophilic groups; this likely occurs through both hydrolysis and deprotonation of silanol groups to form the more nucleophilic silanolate group. A proposed mechanistic route is discussed in the Supporting Information.
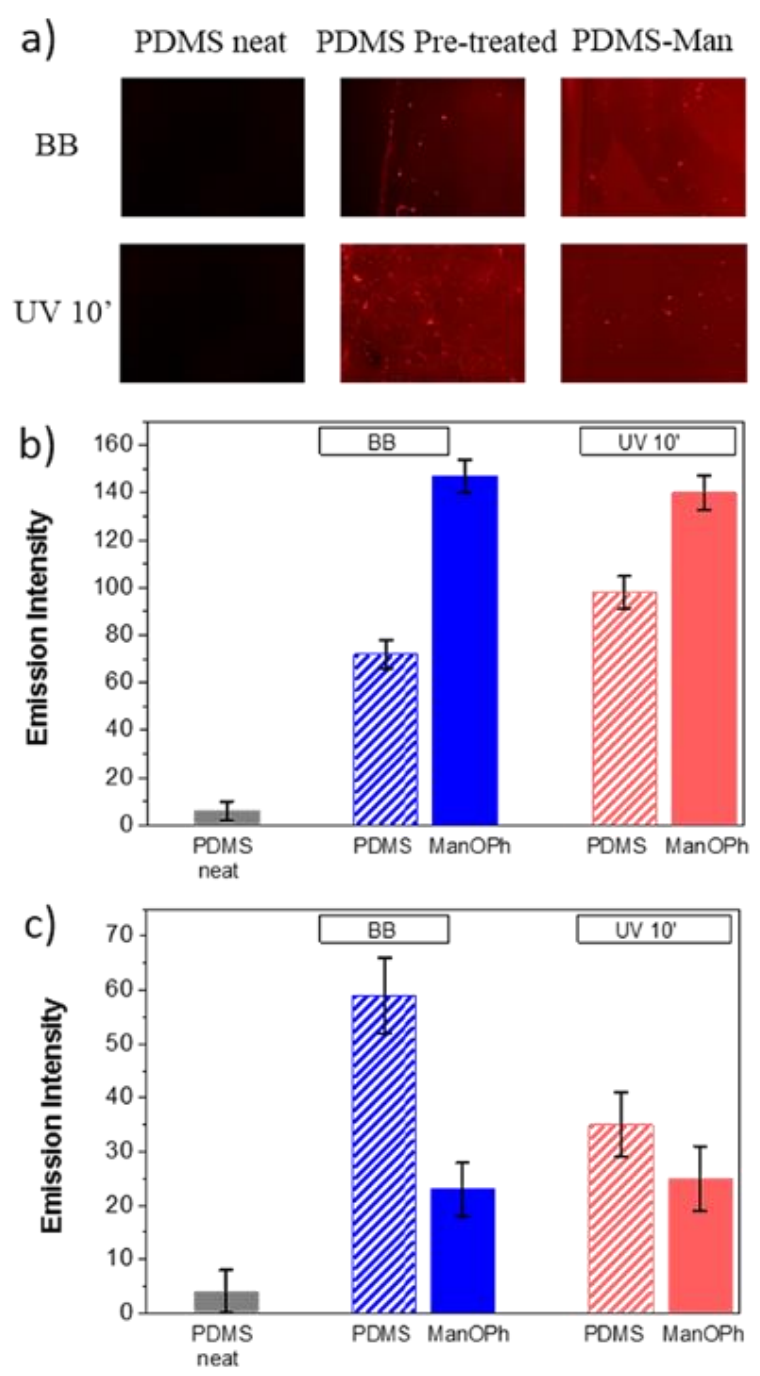

Figure 3: (a) Fluorescence images of neat PDMS, PDMS surfaces pretreated with BB and UV $24410 \mathrm{~min}$ and PDMS coated with ManOPh after binding with ConA. (b) Summary of average emission intensities measured for surface adsorbed ConA on neat PDMS, BB-treated PDMS, PDMS treated with BB and subsequently coated with ManOPh, PDMS treated 10 min under UV light, UV-treated PDMS subsequently coated with ManOPh. (c) Summary of average emission intensities of surface adsorbed BSA on neat PDMS, BB-treated PDMS, PDMS treated with $\mathrm{BB}$ and subsequently coated with $\mathrm{ManOPh}$, PDMS treated 10 min under UV light, UVtreated PDMS subsequently coated with ManOPh. Error bars represent standard deviations calculated over three samples. 
252 In order to confirm that the increase in emission is due to specific mannoside-lectin interactions,

253 we carried out the same experiments with fluorescently labeled BSA, a protein that does not 254 display specific binding with mannoside groups. Figure 3c shows a summary of average 255 emission intensity values obtained using BSA after identical sample treatments. After oxidation 256 via either BB or UV the amount of adsorbed protein increases, as was the case with ConA. 257 However, after functionalization with mannose groups a remarkable decrease in emission is 258 observed, thus indicating that less BSA adsorbs at PDMS-Man surfaces. This indicates, first, 259 that the increase in fluorescence observed for PDMS-Man after incubation in ConA solutions is 260 the result of specific Man-ConA interactions. Second, that immobilization of small saccharides 261 leads to a dcrease in unspecific protein binding compared to oxidized PDMS surfaces, in 262 agreement with observations on the effect of glycoside coatings on polyethersulfones and carbon reported by our group [24, 25, 54], and on PDMS as reported by Zare and co-workers [55].

To further confirm that specific lectin binding interactions are observed after immobilization of glycans via aryldiazonium chemistry, experiments were also carried out using FITC conjugates of ConA and PNA, which display affinity for mannosides and galactosides, respectively [50, 51]. $\mathrm{ManOPh}$ and $\mathrm{GalOPh}$ reacted surfaces were incubated in solutions of both ConA and PNA and the emission intensity was measured to compare protein binding at the two surfaces. Figure 4a shows the fluorescence images in top view while Figure $4 \mathrm{~b}$ shows a comparison of the emission intensities measured under identical conditions. Results indicate that FITC emission reflects the expected binding pattern of the lectins: greater emisison is observed on Man-PDMS surfaces when exposed to ConA, while greater emission is observed on Gal-PDMS surfaces when exposed to PNA. 

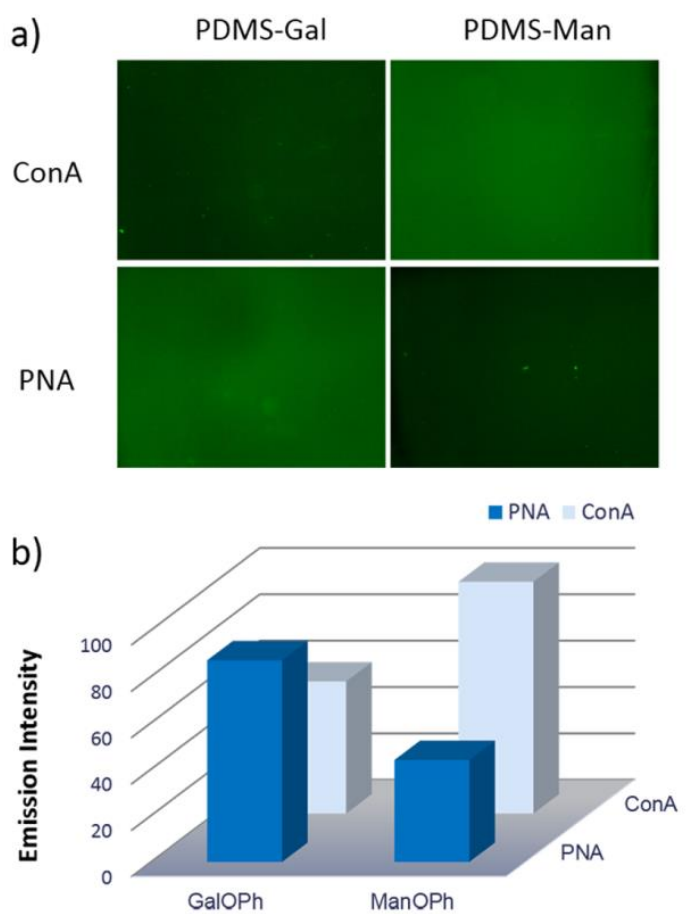

276 Figure 4: (a) Fluorescence images of PDMS coated with GalOPh and ManOPh after incubation 277 in solutions of FITC conjugates of ConA and PNA. (b) Summary of average emission 278 intensities obtained from the images in (a).

Water contact angles of neat PDMS, PDMS functionalized with mannose and PDMS functionalized with lactose for both $\mathrm{BB}$ and UV pre-treatments are shown in Figure 5; the contact angle for neat PDMS is represented by a dashed line. Both pre-treatments increase the hydrophilicity of the PDMS as previously discussed: BB pre-treatment produces the smallest decrease in WCA while 30 min exposure to UV produces the largest one. Functionalization leads to a further decrease in WCA for BB-treated and 10 min UV treated samples. However, for $30 \mathrm{~min}$ of UV exposure, functionalization with glycans was not found to lead to greater hydrophilicity. This is likely due to the high density of oxidized sites present at the PDMS surface after prolongued exposure to UV. Once the density of oxidized sites at the PDMS surface becomes greater than that required for functionaliztaion with bulky glycosides, then no further enhancement of hydrophilicity can be expected to result from the functionalization step. Interestingly, WCA values obtained after BB followed by functionalization are comparable to 

oxidation methods.

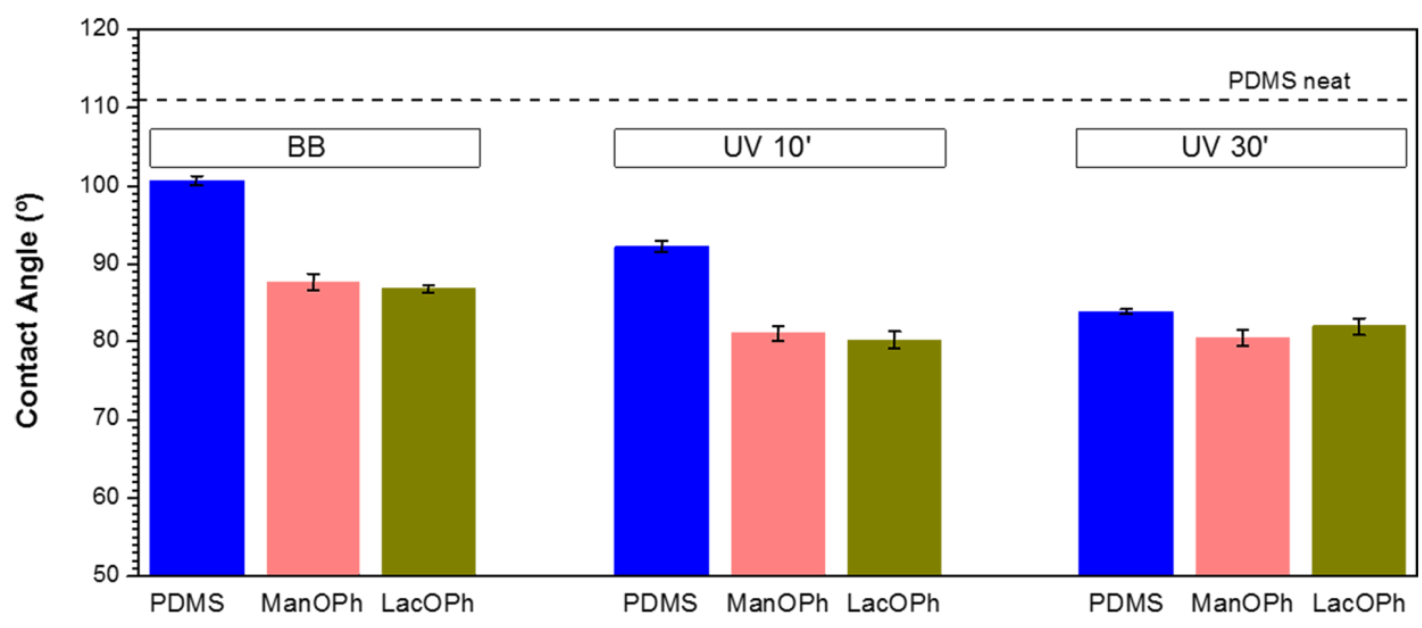

Figure 5: Contact angle of: PDMS, PDMS coated with mannose and PDMS coated with lactose for the different pretreatments: BB, UV10' and UV30'. Dash line: neat PDMS. Error bars represent standard deviations calculated over three samples.

The stability of the modified surfaces obtained via BB pre-treatment was assessed via WCA measurements as a function of time. Figure 6 shows WCA values over 14 days for PDMS surfaces stored under both ambient conditions and under water. No contact angle recovery was observed for either mannoside- or lactoside-functionalized layers after 5 days, and no recovery is observed after 2 weeks when functionalized samples are stored in water. It is worth noting that even the recovery under dry conditions is relatively small, with an increase in WCA of $3.6 \%$ and $5.4 \%$ for mannose and for lactose, respectively, with respect to the initial WCA value. 


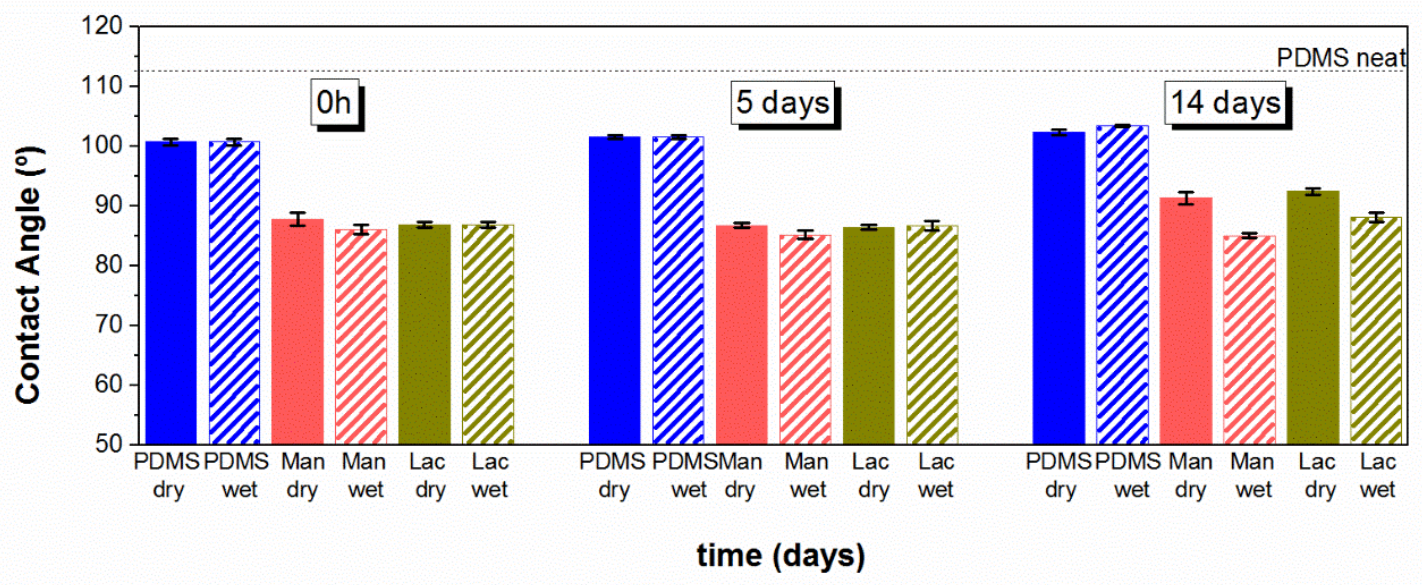

308

309 Figure 6: Contact angle of neat PDMS, PDMS oxidazed with BB, PDMS pre-treated with BB 310 and then coated with mannose or lactose for 0 h, 5 days and 14 days. Spare bars stand for 311 storage in air (dry) and solid bars stand for storage in distillated water (wet). Dash line: neat 312 PDMS. Error bars represent standard deviations calculated over three samples.

\section{Conclusions}

315 Surface modification of PDMS via a two-step method consisting of activation followed by reaction with aryldiazonium cations was demonstrated through a combination of surface spectroscopy, protein binding and contact angle experiments. Results demonstrate that a pretreatment via base/bleach (BB), solutions is sufficient to create reactive sites for aryldiazonium attachment. Aryldiazonium cations bearing glycosides were shown to yield PDMS surfaces with saccharide moieties whose presentation makes them available to specific lectin-glycan binding interactions at the polymer-solution interface, while in the absence of specific binding interactions, surface-bound saccharides led to a reduction in albumin adsorption. Furthermore, glycoside immobilization was found to significantly increase surface hydrophilicity, yielding surfaces with wetting properties similar to those obtained via dry oxidation methods. Studies of surface recovery via water contact angle importantly show that the wetting properties of these functional surfaces are remarkably stable over at least two weeks, especially when stored under wet conditions. 
In summary, functionalization with glycans via aryldiazonium reactions offers a mild and eficient method of obtaining highly hydrophilic and/or biologically functional PDMS surfaces that display good long term stability via a solution based protocol applicable to internal or inaccessible surfaces of channels and devices. Immobilized glycans can be leveraged to either modulate protein binding or tailor surface wettability, therefore this method offers a novel route for modulating PDMS interactions with cells and organisms, an important implication given the multifaceted applications of PDMS in biomedical devices, tissue scaffolds and microfluidic chips.

\section{Acknowledgements}

This publication has emanated from research conducted with the financial support of Enterprise Ireland under Grant Number EI-IP.2014.0307 and of Science Foundation Ireland under Grant Number 12/RC/2278. GC also acknowledges support from Science Foundation Ireland under Grant Number 13/CDA/2213. The authors are thankful to Dr. T. Woods and Dr. B. Ramesh and Prof. J. Boland for access to instrumentation.

\section{REFERENCES}

[1]. L. Luting and T.J. Webster. Preparation of Nanotubular PDMS/PVC Molds for Reduced Catheter Inflammation and Infection. Bioengineering Conference (NEBEC), 2013 39th Annual Northeast, 2013. pp. 74-75.

[2]. J. Curtis and P. Klykken. A comparative assessment of three common catheter materials: Dow Corning Corp, 2008.

[3]. H. Makamba, Y.-Y. Hsieh, W.-C. Sung, and S.-H. Chen, Stable Permanently Hydrophilic Protein-Resistant Thin-Film Coatings on Poly(dimethylsiloxane) Substrates by Electrostatic Self-Assembly and Chemical Cross-Linking, Anal. Chem. 77 (13) (2005) 3971-3978.

[4]. B. Wang, L. Chen, Z. Abdulali-Kanji, J.H. Horton, and R.D. Oleschuk, Aging Effects on Oxidized and Amine-Modified Poly(dimethylsiloxane) Surfaces Studied with Chemical Force Titrations: Effects on Electroosmotic Flow Rate in Microfluidic Channels, Langmuir 19 (23) (2003) 9792-9798. 
[5]. D. Bodas and C. Khan-Malek, Hydrophilization and hydrophobic recovery of PDMS by oxygen plasma and chemical treatment-An SEM investigation, Sens. Actuators, B 123 (1) (2007) 368-373.

[6]. A. Mata, A. Fleischman, and S. Roy, Characterization of Polydimethylsiloxane (PDMS) Properties for Biomedical Micro/Nanosystems, Biomed Microdevices 7 (4) (2005) 281-293.

[7]. D.C. Duffy, J.C. McDonald, O.J.A. Schueller, and G.M. Whitesides, Rapid Prototyping of Microfluidic Systems in Poly(dimethylsiloxane), Anal. Chem. 70 (23) (1998) 4974-4984.

[8]. J.L. Fritz and M.J. Owen, Hydrophobic Recovery of Plasma-Treated Polydimethylsiloxane, J. Adhes. 54 (1-4) (1995) 33-45.

[9]. J.Y. Lai, Y.Y. Lin, Y.L. Denq, S.S. Shyu, and J.K. Chen, Surface modification of silicone rubber by gas plasma treatment, J. Adhes. Sci. Technol. 10 (3) (1996) 231-242.

[10]. K. Efimenko, W.E. Wallace, and J. Genzer, Surface Modification of Sylgard184 Poly(dimethyl siloxane) Networks by Ultraviolet and Ultraviolet/Ozone Treatment, J. Colloid Interface Sci. 254 (2) (2002) 306-315.

[11]. H. Hillborg and U.W. Gedde, Hydrophobicity recovery of polydimethylsiloxane after exposure to corona discharges, Polymer 39 (10) (1998) 1991-1998.

[12]. J. Kim, M.K. Chaudhury, M.J. Owen, and T. Orbeck, The Mechanisms of Hydrophobic Recovery of Polydimethylsiloxane Elastomers Exposed to Partial Electrical Discharges, J. Colloid Interface Sci. 244 (1) (2001) 200-207.

[13]. Y. Liu, J.C. Fanguy, J.M. Bledsoe, and C.S. Henry, Dynamic coating using polyelectrolyte multilayers for chemical control of electroosmotic flow in capillary electrophoresis microchips, Anal. Chem. 72 (24) (2000) 5939-5944.

[14]. S. Hu, X. Ren, M. Bachman, C.E. Sims, G.P. Li, and N. Allbritton, Surface modification of poly(dimethylsiloxane) microfluidic devices by ultraviolet polymer grafting, Anal. Chem. 74 (16) (2002) 4117-4123.

[15]. A. Papra, A. Bernard, D. Juncker, N.B. Larsen, B. Michel, and E. Delamarche, Microfluidic networks made of poly(dimethylsiloxane), Si, and Au coated with polyethylene glycol for patterning proteins onto surfaces, Langmuir 17 (13) (2001) 4090-4095.

[16]. D. Xiao, H. Zhang, and M. Wirth, Chemical modification of the surface of poly(dimethylsiloxane) by atom-transfer radical polymerization of acrylamide, Langmuir 18 (25) (2002) 9971-9976.

[17]. J. Lahann, M. Balcells, H. Lu, T. Rodon, K.F. Jensen, and R. Langer, Reactive polymer coatings: A first step toward surface engineering of microfluidic devices, Anal. Chem. 75 (9) (2003) 2117-2122. 
[18]. B.E. Slentz, N.A. Penner, and F.E. Regnier, Capillary electrochromatography of peptides on microfabricated poly(dimethylsiloxane) chips modified by cerium(IV)-catalyzed polymerization, J. Chromatogr., A 948 (1-2) (2002) 225233.

[19]. H. Mao, T. Yang, and P.S. Cremer, Design and characterization of immobilized enzymes in microfluidic systems, Anal. Chem. 74 (2) (2002) 379-385.

[20]. T. Yang, O.K. Baryshnikova, H. Mao, M.A. Holden, and P.S. Cremer, Investigations of bivalent antibody binding on fluid-supported phospholipid membranes: The effect of hapten density, J. Am. Chem. Soc. 125 (16) (2003) 4779-4784.

[21]. J.N. Lee, C. Park, and G.M. Whitesides, Solvent Compatibility of Poly(dimethylsiloxane)-Based Microfluidic Devices, Anal. Chem. 75 (23) (2003) 6544-6554.

[22]. J.A. Vickers, M.M. Caulum, and C.S. Henry, Generation of Hydrophilic Poly(dimethylsiloxane) for High-Performance Microchip Electrophoresis, Anal. Chem. 78 (21) (2006) 7446-7452.

[23]. C. Donzel, M. Geissler, A. Bernard, H. Wolf, B. Michel, J. Hilborn, and E. Delamarche, Hydrophilic Poly(dimethylsiloxane) Stamps for Microcontact Printing, Adv. Mater. (Weinheim, Ger.) 13 (15) (2001) 1164-1167.

[24]. M.D. Angione, T. Duff, A.P. Bell, S.N. Stamatin, C. Fay, D. Diamond, E.M. Scanlan, and P.E. Colavita, Enhanced Antifouling Properties of Carbohydrate Coated Poly(ether sulfone) Membranes, ACS Appl. Mater. Interfaces 7 (31) (2015) 17238-17246.

[25]. D.R. Jayasundara, T. Duff, M.D. Angione, J. Bourke, D.M. Murphy, E.M. Scanlan, and P.E. Colavita, Carbohydrate Coatings via Aryldiazonium Chemistry for Surface Biomimicry, Chem. Mater. 25 (20) (2013) 4122-4128.

[26]. R.J. Cullen, D.R. Jayasundara, R.J. Baker, G. O' Connell, T. Donnelly, K.E. Ballantine, J.G. Lunney, and P.E. Colavita, Laser-driven rapid functionalization of carbon surfaces and its application to the fabrication of fluorinated adsorbers, RSC Advances 6 (86) (2016) 82924-82932.

[27]. J. Arnhold, S. Mueller, K. Arnold, and E. Grimm, Chemiluminescence intensities and spectra of luminol oxidation by sodium hypochlorite in the presence of hydrogen peroxide, J. Biolumin. Chemilumin. 6 (3) (1991) 189-192.

[28]. M. Kaur and G.K. Bhullar, Partial Characterization of Tamarind (Tamarindus indica L.) Kernel Starch Oxidized at Different Levels of Sodium Hypochlorite, Int. J. Food Prop. 19 (3) (2016) 605-617.

[29]. D. Kuakpetoon and Y.J. Wang, Characterization of different starches oxidized by hypochlorite, Starch/Staerke 53 (5) (2001) 211-218. 
[30]. J.H. Ramsden, R.S. Drago, and R. Riley, A kinetic study of sulfide oxidation by sodium hypochlorite using phase-transfer catalysis, J. Am. Chem. Soc. 111 (11) (1989) 3958-3961.

[31]. Y.-J. Wang and L. Wang, Physicochemical properties of common and waxy corn starches oxidized by different levels of sodium hypochlorite, Carbohydr. Polym. 52 (3) (2003) 207-217.

[32]. H. Yoon and C.J. Burrows, Catalysis of alkene oxidation by nickel salen complexes using sodium hypochlorite under phase-transfer conditions, J. Am. Chem. Soc. 110 (12) (1988) 4087-4089.

[33]. M. Zhao, J. Li, E. Mano, Z. Song, D.M. Tschaen, E.J.J. Grabowski, and P.J. Reider, Oxidation of primary alcohols to carboxylic acids with sodium chlorite catalyzed by TEMPO and bleach, J. Org. Chem. 64 (7) (1999) 2564-2566.

[34]. G. Ducom, B. Laubie, A. Ohannessian, C. Chottier, P. Germain, and V. Chatain, Hydrolysis of polydimethylsiloxane fluids in controlled aqueous solutions, Water Sci. Technol. 68 (4) (2013) 813-820.

[35]. A. Oku, W. Huang, and Y. Ikeda, Monomer recycling for vulcanized silicone rubbers in the form of cyclosiloxane monomers. Role of acid buffers, Polymer 43 (26) (2002) 7289-7293.

[36]. A. Kupareva, J. Wärnå, H. Grénman, and D.Y. Murzin, Transformation of tetramethyldisiloxane in used oil alkali treatment conditions: mechanism and kinetic modeling, J. Chem. Technol. Biotechnol. 91 (1) (2016) 105-112.

[37]. T.C. Kendrick, B. Parbhoo, and J.W. White. Siloxane polymers and copolymers. In: The silicon-heteroatom bond; Armitage DA, Corriu RJP, Kendrick TC, Parbhoo B, Tilley TD, White JW, and Young JC editors. Chichester: John Wiley and Sons, 1991. pp. 67-140.

[38]. M. Ouyang and C.M. Chance, Conductive polymer composites prepared by polypyrrole-coated poly(vinyl chloride) powder: relationship between conductivity and surface morphology, Polymer 39 (10) (1998) 1857-1862.

[39]. B. Schnyder, T. Lippert, R. Kötz, A. Wokaun, V.M. Graubner, and O. Nuyken, UV-irradiation induced modification of PDMS films investigated by XPS and spectroscopic ellipsometry, Surf. Sci. 532-535 (2003) 1067-1071.

[40]. Y. Berdichevsky, J. Khandurina, A. Guttman, and Y.H. Lo, UV/ozone modification of poly(dimethylsiloxane) microfluidic channels, Sens. Actuators, B 97 (2-3) (2004) 402-408.

[41]. A. Oláh, H. Hillborg, and G.J. Vancso, Hydrophobic recovery of UV/ozone treated poly(dimethylsiloxane): adhesion studies by contact mechanics and mechanism of surface modification, Appl. Surf. Sci. 239 (3-4) (2005) 410-423.

[42]. M. Ebara, J.M. Hoffman, P.S. Stayton, and A.S. Hoffman, Surface modification of microfluidic channels by UV-mediated graft polymerization of non-fouling and 'smart' polymers, Radiat. Phys. Chem. 76 (8-9) (2007) 1409-1413. 
[43]. V.-M. Graubner, R. Jordan, O. Nuyken, B. Schnyder, T. Lippert, R. Kötz, and A. Wokaun, Photochemical Modification of Cross-Linked Poly(dimethylsiloxane) by Irradiation at $172 \mathrm{~nm}$, Macromolecules 37 (16) (2004) 5936-5943.

[44]. M.I. Butrón-García, J.A. Jofre-Reche, and J.M. Martín-Martínez, Use of statistical design of experiments in the optimization of $\mathrm{Ar}-\mathrm{O} 2$ low-pressure plasma treatment conditions of polydimethylsiloxane (PDMS) for increasing polarity and adhesion, and inhibiting hydrophobic recovery, Appl. Surf. Sci. 332 (2015) 1-11.

[45]. J.A. Jofre-Reche, J. Pulpytel, H. Fakhouri, F. Arefi-Khonsari, and J.M. MartínMartínez, Surface Treatment of Polydimethylsiloxane (PDMS) with Atmospheric Pressure Rotating Plasma Jet. Modeling and Optimization of the Surface Treatment Conditions, Plasma Processes Polym. (2015) 459-469.

[46]. D.A. Loy. Sol-Gel Processing of Hybrid Organic-Inorganic Materials Based on Polysilsesquioxanes. In: Hybrid Materials; Kickelbick G editor. Weinheim: Wiley-VCH, 2007. pp. 225-254.

[47]. P. Zheng and T.J. McCarthy, A Surprise from 1954: Siloxane Equilibration Is a Simple, Robust, and Obvious Polymer Self-Healing Mechanism, J. Am. Chem. Soc. 134 (4) (2012) 2024-2027.

[48]. D. Cai, A. Neyer, R. Kuckuk, and H.M. Heise, Raman, mid-infrared, nearinfrared and ultraviolet-visible spectroscopy of PDMS silicone rubber for characterization of polymer optical waveguide materials, J. Mol. Struct. 976 (13) (2010) 274-281.

[49]. A. Herrera-Gómez, G. Velázquez-Cruz, and M.O. Martín-Polo, Analysis of the water bound to a polymer matrix by infrared spectroscopy, J. Appl. Phys. 89 (10) (2001) 5431-5437.

[50]. K.A. Maupin, D. Liden, and B.B. Haab, The fine specificity of mannose-binding and galactose-binding lectins revealed using outlier motif analysis of glycan array data, Glycobiology 22 (1) (2012) 160-169.

[51]. N. Sharon and H. Lis, Legume lectins--a large family of homologous proteins, FASEB J. 4 (14) (1990) 3198-3208.

[52]. R.E. Baier, Surface behaviour of biomaterials: The theta surface for biocompatibility, J. Mater. Sci.: Mater. Med. 17 (11) (2006) 1057-1062.

[53]. A.G. Nurioglu, A.C.C. Esteves, and G. de With, Non-toxic, non-biocide-release antifouling coatings based on molecular structure design for marine applications, J. Mater. Chem. B 3 (32) (2015) 6547-6570.

[54]. F. Zen, M.D. Angione, J.A. Behan, R.J. Cullen, T. Duff, J.M. Vasconcelos, E.M. Scanlan, and P.E. Colavita, Modulation of protein fouling and interefacial properties at carbon surfaces via immobilization of glycans using aryldiazonium chemistry, Sci. Rep. 6 (2016) 24840. 
512 [55]. B. Huang, H. Wu, S. Kim, and R.N. Zare, Coating of poly(dimethylsiloxane) with n-dodecyl-[small beta]-d-maltoside to minimize nonspecific protein adsorption, Lab Chip 5 (10) (2005) 1005-1007. 